\title{
REFRAME: Resilience training for GPS
}

W

e need more GPs in the UK if we are to manage our ageing and increasingly multi-morbid population. However, there is a manpower crisis in NHS primary care, with GP recruitment falling and older doctors taking early retirement. Although there has been considerable research into medical staff stress, there are relatively few studies of coping and resilience in doctors. However, there is some evidence that intensive, brief resilience training can be beneficial. Our aim was to establish if REFRAME could help GPs develop skills that improve their well-being and resilience and to establish whether participants feel the outcome measures employed capture their experience.

\section{The GP curriculum and resilience}

Core statement 1: Being a GP requires GPs to:

- Demonstrate compliance with accepted codes of professional practice, showing awareness of your own values and attitudes and how these affect your behaviour

- Demonstrate an approach that shows curiosity, diligence and caring in your encounters with patients and carers

- Promote an organisational culture in which your health and resilience, as well as those of colleagues and staff, is valued and supported

Current workforce planning suggests that it could take up to 30 years for GP numbers to recover to optimum levels (Roberts, 2015). So how can GPs manage the stress of working in an under-staffed primary care system? There is no single, agreed definition of resilience (Balme, Gerada, \& Page, 2015), although it can be usefully thought of as the capacity to bounce back from stress or adversity (Carver, 1998). Resilience is not the solution to the problems in primary care, a much wider political solution is needed, but it has been proposed as a means to help protect individuals, so that they can continue to function and thrive despite the intense pressures that they are under.

Does resilience training work? There is some evidence that it is effective. For instance, research exploring the impact of a 90-minute Stress Management and Resilience Training (SMART) designed for doctors observed significant improvements at 8-week follow-up in resilience, perceived stress, anxiety and quality of life (Sood, Prasad, Schroeder, \& Varkey, 2011).

REFRAME is a 150-minute resilience training programme that was initially designed for first-year foundation doctors and subsequently modified for GPs (Box 1). Our aim was to evaluate whether this would be acceptable to GPs and relevant to the problems that concern them. This article explores this issue and presents our findings to date.

\section{What did we do?}

The REFRAME course took place in Southampton and was offered by the Southampton GP Education Unit. As this was an evaluation of an educational programme, no ethical approval was required. All attendees were invited to participate. The key questions that we explored are outlined in Box 2.

\section{Evaluation measures}

In order to evaluate the programme we employed the Westminster Quantitative Feedback Questionnaire, the Warwick-Edinburgh Mental Well-Being Scale, and a series of open-ended free-text questions. 


\section{Box 1. The core components of REFRAME.}

\section{Learning aims}

- Knowledge about individual and organisational stressors in medicine

- Understanding of neurobiology of resilience, empathy and burnout

\section{Learning outcomes}

- To explore the individual's experience of the effects of stress in the workplace

- To gain insights and techniques to maintain mental well-being

- To feel empowered to improve working life and thus self and patient care

\section{Reflections}

- How resilient do we feel? - WORKSHOP

- Why now? The stresses of clinical work PRESENTATION

- Clinician stress: impact on patients and colleagues - WORKSHOP

- Reflections on self-management and lifestyle WORKSHOP

\section{Realities}

- Evolution and emotions - PRESENTATION

- Neurobiology of stress and resilience PRESENTATION

- Survival instincts, empathy and communication PRESENTATION

- Burnout and how to recognise it - WORKSHOP

\section{Resources}

- Regulating your autonomic nervous system: Can it be done? - DEMO/EXPERIENTIAL

- Building resilience and managing our own stress WORKSHOP

- Your Resilience Matrix - WORKSHOP

- Setting goals for taking care of ourselves WORKSHOP

\section{Experience of skills and their impact}

- Mindfulness

- Heart rate variability biofeedback

- Regulated breathing

- Positive emotion

\section{Reflection on}

- Resilience and stressors

- Burnout

- Your biofeedback data showing stress response and recovery

- Your own 'Resilience Matrix'

- Your colleagues' experiences

- Your SMART goals for resilience-building

The Westminster Quantitative Feedback Questionnaire is a six-item measure of satisfaction with a course. Participants are asked to rate statements such as: 'The workshop was useful to me' and 'The pace of the day

\section{Box 2. Key research questions.}

- Do the evaluations we propose capture participants' experience of the course and its impact?

- Does the course attract GPs and do they embrace it and participate?

- Based on the evaluations used in the pilot does the course improve the well-being and resilience of GPs?

- How can the course be improved to fulfil the needs of GPs?

- What sort of online resources would participants value?

was just right' on a five-point Likert scale. The questionnaire was expanded to include three open-ended free-text questions that were used to evaluate participants' experiences of the workshop:

- 'Please tell us what made you come on this course, what you liked about it and how we could improve it

- 'Were your learning needs addressed with this course and what exactly did you learn'

- 'Will you be doing anything differently in your job, either short or long term, as a result of this course'

The Warwick-Edinburgh Mental Well-Being Scale (WEMWBS) is a 14-item measure of well-being (Tennant et al., 2007). It includes statements such as: 'I've been feeling optimistic about the future' and 'I've had energy to spare', which participants are asked to rate on five-point Likert scale. Scores range from 14 to 70, with higher scores indicating better mental well-being. The WEMWBS is particularly suitable for mental health promotion activities where negatively worded questionnaires may give participants the sense that they are attending something intended for people who are unwell.

A series of open-ended free-text questions were also developed to explore participants' experiences and perceptions of the workshop and its impact at 2-week follow-up (T2). These included:

- If we had online material, what should be included?

- If you have any questions about the questionnaires, or can suggest more suitable ones, please do so

- If you have any comments about the outcome of attending the resilience course and any impact it may have had on your work or personal life, please outline them

- Do you have any other comments about the course and its impact?

\section{Procedure used}

All REFRAME attendees invited to participate in the research were given an information sheet outlining the purpose of the research and asked to sign a consent form if they wished to participate. Participants were asked to complete a hard copy of the WEMWBS and the Westminster Quantitative Feedback Questionnaire at 
the end of the session (baseline data). Two weeks later (T2), participants were emailed a link to an online survey that included the WEMWBS and the T2 open-ended free-text questions. One reminder email was sent to all participants. Six weeks after completing the workshop (T3), participants were sent another link to complete the WEMWBS for a third time. As at T2, one reminder email was sent to all participants.

Descriptive statistics were generated for the WEMWBS data. A simple thematic analysis was conducted on the open-ended free-text questions by the first author using the approach outlined by Braun and Clarke (2006).

\section{What did we find?}

There were 15 participants in the study. Initially 20 people said they would attend with five cancelling at the last minute for work-related reasons. The mean age was 46 years, with a range between 32 and 57 years.

\section{Why did participants attend?}

The majority of the doctors who attended said they were looking for more information about resilience, either in terms of tools they could use, or information about resilience in organisations (Box 3). A third of GPs reported attending because they were feeling very stressed or burnt out and were looking for a way to cope. It is noteworthy that two participants mentioned that they chose to attend to develop resilience as a consequence of complaints made against them. This implies that a substantial minority of GPs have concerns about their own health and ability to cope with workplace demands.

\section{Box 3. Quotes: Attendance.}

- I am increasingly feeling physically stressed at my practice so I want to cope with this better. So it has an impact on me personally and to help my colleagues, especially the younger ones

- I am on sabbatical to try and avoid burnout, and this course seemed appropriate

- I am upset with myself in reaction to a complaint i.e. how I felt physically and emotionally

The GPs who participated in this pilot were looking for practical tools that they could apply immediately to help them and their colleagues. The impression gained was of a group who, though their coping resources were depleted, were trying to find ways of carrying on in their work.

\section{Course feedback}

The immediate post-course feedback, indicated that the majority of the participants agreed or strongly agreed that that REFRAME was useful, clearly communicated, delivered at the right pace, and with a good balance of theory and practice. All of the GPs found the areas covered useful or very useful, and $93 \%$ said they planned to use the suggested approaches to build resilience.

\section{Was there a measurable improvement in GP well-being?}

The mean WEMWBS score at baseline was 50.13 (SD 6.16, range 35-58 with higher scores indicating better well-being), which represents the norm for this population (Davidson, Sewel, Tse, \& O'Connor, 2009). However, the wide range of scores suggests there may be important differences in the well-being of participants.

Looking at individual questions on the WEMWBS, many of the participants at baseline felt that they rarely had any energy to spare (number $(N)=6$ ) or felt relaxed $(N=3)$. A small increase in the WEMWBS mean scores was observed for the six participants who completed the WEMWBS at both T1 (mean $(M)=48.50$, standard deviation $(S D)=7.71)$ and $T 2(M=49.67, S D=7.00)$, suggesting a slight increase in well-being. There were only two responses at 6 weeks and these indicated a small improvement over time.

\section{Gains from REFRAME}

The participants said they took away an increased understanding of resilience, as well as tools to support resilience in their lives (Box 4). They garnered more knowledge on the neurobiology of stress and recovery, some re-evaluated their learning needs, generated SMART goals and some said they were clear that they have useful insights that will help them manage their professional lives. The majority planned to make changes that they felt would be valuable to them as individuals. At 2-week follow-up three participants noted that attending the sessions had led them to make small changes in their lives, which has translated into important real-world change.

\section{Box 4. Quotes: Gains from REFRAME.}

- It was useful to articulate difficulties in a constructive environment and it helped me to move on in small ways

- I have put into place several points that I came up with during the session - seemed minor points at the time (e.g. Walk after duty day rather than glass of wine, read non medical material/book), but I have felt more in control and positive in the weeks since

- Excellent to remind me what I should be doing, and lovely to be with like minded people, the difficulty is still time 
What could be improved?

Participants felt that the training was beneficial, although some felt that a whole day of training would be preferable. In the 2-week follow-up questionnaire, participants said they would like to access resilience resources, such as meditation mp3 files, reading lists, animations and the course material. Although participants were keen to have found the shared group experience particularly beneficial (please see Box 5). This suggests that moving forward it would be useful to consider a two-tiered approach, providing additional online resources and training alongside extended face-to-face training (whole days, developing peer-support networks), perhaps using a blended learning approach.

\section{Box 5. Quotes: Improvements.}

- Main benefit was being with other doctors with varied difficulties and sharing solutions. I would not be interested in online materials. Too much time on the computer already

- Download of meditation/relaxation and tips to remind me - book list and links to useful material

- A video showing the slow inhalation and slow expiration was used in the meditation section - it would be cool to find out where to get that access to online material, they also highlighted that they

The range of WEMWBS scores, along with the qualitative data collected, suggests that there may be subgroups of GPs with particularly low levels of well-being. If this is the case, it would be interesting to look further into the impact of age, gender, training and experience, as well as enthusiasm to make changes. It is likely that different training schemes might be needed for those who are most in difficulty.

Given that a quarter of those who had originally signed up for the training were unable to attend due to work demands, there may also be issues around how to make such training accessible. Those hardest pushed may not have been able to attend.

Content, balance and pragmatic usefulness of the 150-minute course was highly rated, as was the impact of the peer-support provided by coming together as a group of professionals to share problems and solutions to the current difficult working conditions. Although followup was limited, there is evidence that participants were able to implement small, but meaningful, changes to their routines, which they felt led to positive changes in their lives.

\section{Conclusions and moving forward}

REFRAME appears to have been appreciated by GPs. It is well-structured, covers appropriate topics and seems to provide relevant resilience tools, which are easy to implement.

Resilience training for GPs has gained popularity in recent years. GPs are seeking solutions to their increased stress levels caused by workload pressures, diminishing resources and rising patient expectations. However, political tension is created, due to organisations seeing resilience as a potential solution to stem the exodus of GPs from the profession either to work abroad, change their working patterns to become locums, reduce their hours, or leave the profession completely. This has led to resilience training being perceived as a 'sticking plaster' to solve a problem that actually requires major surgery.

This evaluation of a pilot course (REFRAME) was in Southampton among a self-selected group of GPs. The numbers are small, and this group may not be representative of GPs in the UK. This does significantly limit the conclusions that can be drawn, but we do feel that this evaluation has provided us with some useful insights.

Although the mean WEMWBS was normal, some individual GPs attending the course reported that they were stressed and burnt out and were looking for tools to help them better cope. There was no demonstrable change in WEMWBS as a result of the course, but the numbers completing follow-up were very small (5) and this limits any clear conclusions.
The use of resilience training, REFRAME in particular, will not solve the problems of the NHS. It may help individuals to maintain their own health and well-being, function better, and survive as GPs while the major political changes needed to sustain the NHS take place.

Further work is needed to establish if this is the right approach. We would not deliver treatment to our vulnerable patients without good evidence and the same ethics and requirements should underpin interventions for vulnerable GPs. Formal focus groups with GPs, held in conjunction with the RCGP, are currently under way and will 
be used to further to develop these workshop in the future.

\section{Key points}

- There is a current crisis in GP numbers and widespread burnout

- Intensive, brief resilience training seems to be beneficial

- REFRAME resilience training for GPs seems to be appreciated and well-received

- Findings from this study and follow-up work will improve the delivery and evaluation of REFRAME

\section{References and further information}

- Balme, E., Gerada, C., \& Page, L. (2015). Doctors need to be supported, not trained in resilience. Retrieved from http://careers.bmj.com/careers/ advice/Doctors_need_to_be_supported,_not_ trained_in_resilience

- Braun, V., \& Clarke, V. (2006). Using thematic analysis in psychology. Qualitative Research in Psychology, 3(2), 77-101. doi: 10.1191/ 1478088706qp063oa

- Carver, C. S. (1998). Resilience and thriving: Issues, models, and linkages. Journal of Social Issues, 54, 245-266

- Davidson, S., Sewel, K., Tse, D., \& O'Connor, R. (2009). Well? What do you think: The fourth

\section{Dr Siobhan Lynch}

University of Southampton and University of Westminster

Email: glewith@scmrt.org.uk

\section{Dr Mark Lown}

University of Southampton

Ms Daghni Rajasingami

GSTT Trust and Faculty of Medical Leadership

Professor David Peters, Professor Damien Ridge, Dr Anna Cheshire and Ms Kate Fismer University of Westminster

\section{Professor Sarah Stewart-Brown}

University of Warwick

\section{Dr Henry Lewith}

Anaesthetic Registrar, Oxford Deanery

\section{Dr Olivia Jagger}

Faculty of Medical Leadership and ACF Wessex GP Deanery

\section{Professor George Lewith}

University of Southampton and University of Westminster national Scottish survey of public attitudes to mental well-being and mental health problems. Retrieved from www.gov.scot/Publications/2009/ 09/15120147/8

- Lown, M., Lewith, G., Simon, C., \& Peters, D. (2015). Resilience: What is it, why do we need it, and can it help us? British Journal of General Practice, 65(639), e708-e710. doi: 10.3399/ bjgp15X687133

- RCGP. Core statement 1: Being a GP. Retrieved from www.rcgp.org.uk/training-exams/gp-curriculum-overview/online-curriculum/1-being-a-gp.aspx

- Roberts, N. (2015). Election pledges on GP recruitment may take 30 years to deliver, RCGP warns. Retrieved from http://www.gponline.com/elec tionpledges-gp-recruitment-may-30-years-deliverrcgp-warns/article/1345748

- Sood, A., Prasad, K., Schroeder, D., \& Varkey, P. (2011). Stress management and resilience training among Department of Medicine faculty: A pilot randomized clinical trial. Journal of General Internal Medicine, 26(8), 858-861

- Tennant, R., Hiller, L., Fishwick, R., Platt, S., Joseph, S., Weich, S., \& Stewart-Brown, S. (2007). The Warwick-Edinburgh mental wellbeing scale (WEMWBS): Development and UK validation. Health and Quality of Life Outcomes, 5(1), 63. doi: 10.1186/1477-7525-5-63 